\title{
Using an Open GIS Framework and Epidemiological Intelligence for Dengue Surveillance
}

\author{
Ta-Chien Chan*1, Bo-Cheng Lin¹, Chiao-Ling Kuo and Li-hsiang Chiang ${ }^{2}$ \\ ${ }^{1}$ Academia Sinica, Taipei City, Taiwan; ${ }^{2}$ Public Health Bureau, Pingtung County Government, Pingtung County, Taiwan
}

\section{Objective}

In this paper we designed one cross-platform surveillance system to assist dengue fever surveillance, outbreak investigation and risk management of dengue fever.

\section{Introduction}

In the 2015 dengue outbreak in Taiwan, 43,784 people were infected and 228 died, making it the nation's largest outbreak ever. Facing the increasing threat of dengue, the integration of health information for prevention and control of outbreaks becomes very important. Based on past epidemics, the areas with higher incidence of dengue fever are located in southern Taiwan. Without a smart and integrated surveillance system, the information on case distribution, high risk areas, mosquito surveillance, flooding areas and so on is fragmented. The first-line public health workers need to check all this information through different systems manually. When outbreaks occurred, paper-based outbreak investigation forms had to be prepared and filled in by public health workers. Then, they needed to enter part of this information into Taiwan CDC's system. Duplicated work occurred and cost lots of labor time during the epidemic period. Therefore, we choose one rural county, Pingtung County, with scarce financial resources, to set up a new dengue surveillance system.

\section{Methods}

We designed a web-based cross-platform system based on an open geographical information system (GIS) framework including Openlayers, Javascript, PHP, MySQL and open data from government open data in Taiwan. There were seven epidemiological intelligence functions within the system including risk management, outbreak investigation, planning controlled areas, intelligent detection of highrisk areas, useful tools for decision making, historical epidemics, and system management. The website was developed by responsive web design which can let public health workers check information and fill in the investigation form by any devices.

\section{Results}

The system was promptly set up in June 2016. With first-line public health workers' efforts and the help of the surveillance system, there were no indigenous dengue fever cases after the system was implemented. There were sporadic imported cases from southeast Asia. The dengue surveillance system achieved three major improvements: integration of all decision support information; digitalization and automation of outbreak investigation; and planning the control areas. The results on outbreak investigation and mosquito surveillance can directly transfer to Taiwan CDC's database by Web Application Programming Interface (API). It can avoid duplicated work for disease surveillance.

\section{Conclusions}

Through introducing the new dengue surveillance system into local health departments, first-line public health workers can update all epidemic information at the same time. During epidemic periods, it can provide demographic, epidemiological, environmental, and entomological information for decision making. During non-epidemic periods, it can highlight the high risk areas for enhanced surveillance to reduce the risk of outbreaks.

Keywords

GIS; Risk management; Outbreak control; Open data; Geocoding

\section{Acknowledgments}

This research was supported by a grant from National Health Research Institute, Taiwan.

\section{References}

Duncombe J, Clements A, Hu W, Weinstein P, Ritchie S, Espino FE: Geographical Information Systems for Dengue Surveillance. The American Journal of Tropical Medicine and Hygiene 2012, 86(5):753755.

Hernández-Ávila JE, Rodríguez M-H, Santos-Luna R, Sánchez-Castañeda V, Román-Pérez S, Ríos-Salgado VH, Salas-Sarmiento JA: NationWide, Web-Based, Geographic Information System for the Integrated Surveillance and Control of Dengue Fever in Mexico. PLoS One 2013, 8(8):e70231.

\author{
*Ta-Chien Chan \\ E-mail: dachianpig@gmail.com
}

